\title{
Ep.6.31
}

EESTI NSV TEADUSTE AKADEEMIA TOIMETISED.

FUUSIKA * MATEMAATIKA

ИЗВЕСТИЯ АКАДЕМИИ НАУК ЭСТОНСКОИ ССР. ФИЗИКА * МАТЕМАТИКА

PROCEEDINGS OF THE ACADEMY OF SCIENCES OF THE ESTONIAN SSR. PHYSICS * MATHEMATICS

$1985,34,1$

УдК $535.391 .4: 535.345 .673$

П. КАРД

\section{НОВЫЙ ВИД ФОРМУЛ ФРЕНЕЛЯ}

\section{Введение}

В настоящей статье нашей целью является вывод формул Френеля в новом виде, отличающемся исключительной простотой. Речь идет о формулах, определяющих амплитуды отраженного и преломленного света. Новые формулы удобны в особенности в применении к теории многослойных интерференционных пленок. Основные из этих применений будут здесь показаны.

Новое представление формул Френеля было введено и использовано уже в $\left[{ }^{1}\right]$, но не нашло до сих пор того внимания, которого оно, по нашему мнению, заслуживает. Мы не будем повторять данного в $\left[{ }^{1}\right]$ вывода, но изложим более простой и прямой способ. Вдобавок мы откажемся от обычного (принятого, в частности, и в $\left[{ }^{1}\right]$ ) условия равенства единице относительной магнитной проницаемости всех сред. Тем самым формулы станут более общими, не в ущерб, однако, их простоте. Но все среды будем считать'в этой статье изотропными и однородными.

\section{Формулы Френеля}

Зависимость полевых величин в монохроматической плоской волне от радиуса-вектора $\mathbf{r}$ и времени $t$ будем выражать множителем $\exp \left[i \omega\left(t-c^{-1} n \mathrm{k}_{0} \mathrm{r}\right)\right]$, где $\omega-$ частота, $c-$ скорость света в вакууме, $n$ - показатель преломления среды, $\mathrm{k}_{0}$ - единичный вектор, определяющий направление волны. Показатель преломления является, вообще говоря, комплексной величиной. Он связан с относительными электрической и магнитной проницаемостями $\varepsilon$ и $\mu$, тоже в общем случае комплексными, известной формулой

$$
n=(\varepsilon \mu)^{1 / 2} \text {. }
$$

Для вектора $\mathbf{k}_{0}$ будем тоже допускать комплексные значения, сохраняя и в этом случае название «плоская волна», так как поверхности равной фазы остаются плоскостями, хотя амплитуда на каждой такой плоскости не постоянна. Обычно волны с комплексным вектором $\mathbf{k}_{0}$ называют неоднородными, но мы не будем пользоваться этим названием.

Напряженности электрического и магнитного полей Е и Н связаны в плоской волне известной формулой

$$
\mathbf{H}=\left(\varepsilon \varepsilon_{0} / \mu \mu_{0}\right)^{1 / 2}\left(k_{0} \times \mathbf{E}\right),
$$

а модули этих векторов - формулой

1 ENSV TA Toimetised. F * M 11985

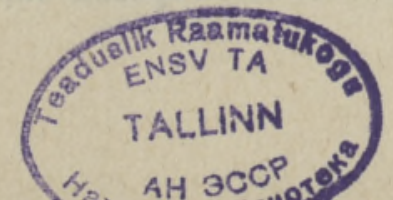




$$
H=\left(\varepsilon \varepsilon_{0} / \mu \mu_{0}\right)^{1 / 2} E,
$$

где $\varepsilon_{0}$ и $\mu_{0}$ - электрическая и магнитная постоянные.

Пусть теперь плоская монохроматическая волна падает на плоскую границу раздела двух сред, отражаясь и преломляясь на этой границе. Среду, откуда волна падает, обозначим индексом 1 , другую среду - индексом 2. Теми же индексами будем обозначать все величины в той или другой среде. Так, угол падения обозначим через $\vartheta_{1}$, угол преломления через $\hat{\vartheta}_{2}$. Оба угла могут иметь комплексные значения. Отраженную волну будем обозначать штрихом.

Запишем граничные условия, состоящие, как известно, в непрерывности тангенциальных комнонентов векторов Е и Н. В случае ТЕ-поляризации (электрический вектор перпендикулярен плоскости падения) граничные условия имеют, с учетом формулы (3), вид

$$
\begin{gathered}
E_{1}+E_{1}^{\prime}=E_{2} \\
\left(\varepsilon_{1} / \mu_{1}\right)^{1 / 2} \cos \vartheta_{1}\left(E_{1}-E_{1}^{\prime}\right)=\left(\varepsilon_{2} / \mu_{2}\right)^{1 / 2} \cos \vartheta_{2} E_{2} .
\end{gathered}
$$

В случае ТМ-поляризации (магнитный вектор перпендикулярен плоскости падения), аналогично,

$$
\begin{gathered}
\left(\varepsilon_{1} / \mu_{1}\right)^{1 / 2}\left(E_{1}+E_{1}^{\prime}\right)=\left(\varepsilon_{2} / \mu_{2}\right)^{1 / 2} E_{2}, \\
\cos \vartheta_{1}\left(E_{1}-E_{1}^{\prime}\right)=\cos \vartheta_{2} E_{2} .
\end{gathered}
$$

В формулах (4) и (5) под $E_{1}, E_{1}^{\prime}$ и $E_{2}$ следует понимать амплитуды, так как переменный множитель сокращается. Чтобы придать этим формулам в случае обеих поляризаций одинаковый вид, введем величины

$$
\begin{aligned}
& v_{\mathrm{TE}}=\frac{1}{2} \ln \left((\varepsilon / \mu)^{1 / 2} \cos \vartheta\right), \\
& v_{\mathrm{TM}}=\frac{1}{2} \ln \left((\varepsilon / \mu)^{-1 / 2} \cos \vartheta\right)
\end{aligned}
$$

H

$$
A=\left(\varepsilon \varepsilon_{0} / \mu \mu_{0}\right)^{1 / 4} \cos ^{1 / 2} \vartheta E=\left(\varepsilon \varepsilon_{0} / \mu \mu_{0}\right)^{-1 / 4} \cos ^{1 / 2} \vartheta H .
$$

Тогда формулы (4) и (5) объединяются в единую запись

$$
\begin{aligned}
& A_{1}+A^{\prime}{ }_{1}=e^{v_{1}-v_{2}} A_{2}, \\
& A_{1}-A^{\prime}{ }_{1}=e^{-\left(v_{1}-v_{2}\right)} A_{2},
\end{aligned}
$$

где зависимость от поляризации учитывается неявным образом через формулы (6). Решая уравнения (8) относительно $A_{1}^{\prime}$ и $A_{2}$, находим

$$
\begin{aligned}
& A^{\prime}{ }_{1}=A_{1} \text { th }\left(v_{1}-v_{2}\right), \\
& A_{2}=A_{1} \operatorname{ch}^{-1}\left(v_{1}-v_{2}\right) .
\end{aligned}
$$

Это и есть новый вид формул Френеля. Подчеркнем вновь, что все входящие в эти формулы величины в общем случае комплексны.

\section{Рефрактор и обобщенная амплитуда}

Величины $v$ и $A$, определяемые формулами (6) и (7), могут показаться искусственными и лишенными прямого физического смысла. Однако их практический смысл выясняется в дальнейших применениях. Величину $v$ мы предлагаем называть рефрактором среды, поскольку она существенным образом определяет свойства вещества как прелом- 
ляющей среды. Необходимо только иметь в виду, что рефрактор зависит не только от самой среды, но и от угла преломления и поляризации. В случае нормального падения формулы (6) определяют рефрактор с точностью до знака. Для определенности присвоим волне в случае нормального падения поляризацию ТЕ, так что в этом случае

$$
v_{\text {норм }}=\frac{1}{4} \ln (\varepsilon / \mu) \text {. }
$$

Исключив $\varepsilon$ и введя взамен показатель преломления, перепишем формулы (6) и (10) в виде

$$
\begin{aligned}
& v_{\mathrm{TE}}=\frac{1}{2} \ln \left(n \mu^{-1} \cos \vartheta\right), \\
& v_{\mathrm{T} M}=\frac{1}{2} \ln \left(n^{-1} \mu \cos \vartheta\right)
\end{aligned}
$$

и

$$
v_{\text {норм }}=\frac{1}{2} \ln \left(n \mu^{-1}\right) \text {. }
$$

В случае $\mu=1$ эти формулы принимают вид

$$
\begin{aligned}
& v_{\mathrm{TE}}=\frac{1}{2} \ln (n \cos \vartheta), \\
& v_{\mathrm{TM}}=\frac{1}{2} \ln \left(n^{-1} \cos \vartheta\right)
\end{aligned}
$$

и

$$
v_{\text {норм }}=\frac{1}{2} \ln n,
$$

т. е. рефрактор при данном угле и поляризации зависит в этом случае только от показателя преломления.

Величину $A$ мы назовем обобщценной амплитудой волны. Для выяснения смысла этого понятия отметим, что в случае вещественности величин $\varepsilon, \mu$ и $\vartheta$

$$
A A^{*}=\cos \vartheta E H^{*} \text {. }
$$

С другой стороны, усредненный по времени модуль вектора Пойнтинга равен в этом случае

$$
S=\frac{1}{2} E H^{*}
$$

Следовательно,

$$
\frac{1}{2} A A^{*}=S \cos \vartheta
$$

т. е. $\frac{1}{2} A A^{*}$ означает поток энергии волны через единичную площадку, взятую на плоскости раздела сред. Таким образом, обобщенная амплйтуда близко связана с потоком энергии. Мы распространяем это название и на общий случай, когда $\varepsilon, \mu$ и $\vartheta$ комплексны, хотя формулы (15) - (17) тогда неприменимы. 


\section{Амплитудные, энергетические и линейные коэффициенты отражения и преломления}

Амплитудными коэффициентами отражения $r$ и преломления $t$ мы называем отношения обобщенных амплитуд. Из формул (9) следует

$$
\begin{aligned}
& r=A_{1}^{\prime} / A_{1}=\operatorname{th}\left(v_{1}-v_{2}\right), \\
& t=A_{2} / A_{1}=\operatorname{ch}^{-1}\left(v_{1}-v_{2}\right) .
\end{aligned}
$$

В случае вещественности проницаемостей сред и углов падения и преломления $r$ и $t$ тоже вещественны. Из формул (17) и (18) вытекают тогда следующие формулы для энергетических коэффициентов отражения и преломления:

$$
\begin{aligned}
& R=r^{2}=\operatorname{th}^{2}\left(v_{1}-v_{2}\right), \\
& T=t^{2}=\operatorname{ch}^{-2}\left(v_{1}-v_{2}\right),
\end{aligned}
$$

причем, как и должно быть,

$$
R+T=r^{2}+t^{2}=1 .
$$

В общем случае, когда проницаемости и углы комплексны, формулы (19) теряют, вообще говоря, точный смысл, хотя приближенный смысл в некоторых случаях сохраняется.

Линейными коэффициентами отражения и преломления мы называем величины

$$
\begin{aligned}
& a=r / t, \\
& b=1 / t .
\end{aligned}
$$

Для них, согласно формулам (18), имеют место выражения

$$
\begin{aligned}
& a=\operatorname{sh}\left(v_{1}-v_{2}\right) ; \\
& b=\operatorname{ch}\left(v_{1}-v_{2}\right),
\end{aligned}
$$

причем, в случае вещественности проницаемостей и углов,

$$
\begin{aligned}
& a^{2}=R / T, \\
& b^{2}=1 / T .
\end{aligned}
$$

Равенство

$$
b^{2}-a^{2}=1,
$$

равносильное в том же случае равенству $R+T=1$, верно и в общем случае, независимо от соотношений (23).

Смысл названия «линейные коэффициенты» выясняется в теории многослойных пленок.

\section{Двустороннее падение}

Рассмотрим ситуацию, когда на одну и ту же плоскую границу раздела двух сред падают, отражаясь и преломляясь, когерентные плоские волны с обеих сторон, причем угол падения в каждой из двух сред равен углу преломления в той же среде (см. рис. 1). В обеих средах имеются тогда по две волны - прямая и обратная. Обозначим обобщенные амплитуды четырех волн через $A_{1}, A_{2}$ (прямые волны) и $A_{1}^{\prime}, A_{2}^{\prime}$ (обратные волны). Тогда, учитывая когерентность всех волн, имеем, согласно формулам (9), соотношения 
Рис. 1.

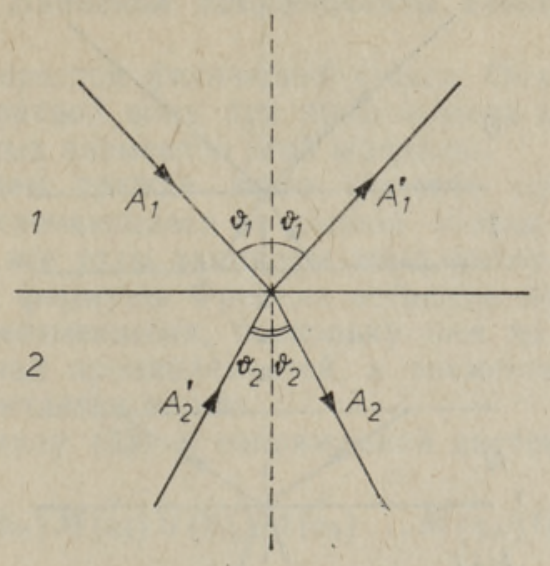

$$
\begin{aligned}
& A^{\prime}{ }_{1}=A_{1} \operatorname{th}\left(v_{1}-v_{2}\right)+A^{\prime}{ }_{2} \operatorname{ch}^{-1}\left(v_{1}-v_{2}\right), \\
& A_{2}=A_{1} \operatorname{ch}^{-1}\left(v_{1}-v_{2}\right)-A^{\prime}{ }_{2} \operatorname{th}\left(v_{1}-v_{2}\right) .
\end{aligned}
$$

Выражая отсюда $A_{1}$ и $A^{\prime}{ }_{1}$, находим

$$
\begin{aligned}
& A_{1}=A_{2} \operatorname{ch}\left(v_{1}-v_{2}\right)+A^{\prime}{ }_{2} \operatorname{sh}\left(v_{1}-v_{2}\right) ; \\
& A^{\prime}{ }_{1}=A_{2} \operatorname{sh}\left(v_{1}-v_{2}\right)+A^{\prime}{ }_{2} \operatorname{ch}\left(v_{1}-v_{2}\right) .
\end{aligned}
$$

Этим формулам удобно придать матричный вид. Обозначим

$$
G(u)=\left(\begin{array}{ll}
\operatorname{ch} u & \operatorname{sh} u \\
\operatorname{sh} u & \operatorname{ch} u
\end{array}\right)
$$

и

$$
\mathrm{A}=\left(\begin{array}{l}
A \\
A^{\prime}
\end{array}\right)
$$

Тогда

$$
\mathrm{A}_{1}=G\left(v_{1}-v_{2}\right) \mathrm{A}_{2} .
$$

Эта формула является матричной записью формул Френеля, обобщенных на случай двустороннего падения света на границу раздела сред. Ее можно написать в несколько ином виде. Так как

$$
G\left(v_{1}-v_{2}\right)=G\left(v_{1}\right) G\left(-v_{2}\right),
$$

To

$$
G\left(-v_{1}\right) \mathrm{A}_{1}=G\left(-v_{2}\right) \mathrm{A}_{2} .
$$

\section{Многослойная пленка}

Многослойную пленку составляют несколько сред, разделенных параллельными плоскостями. Вне пленки находятся ограничивающие полубесконечные среды. Обозначим число слоев пленки через $N$, перенумеруем wх $1,2, \ldots N$ в произвольном направлении, которое назовем прямым, а ограничивающим средам присвоим индексы 0 и $N+1$. Направление, обратное прямому, будем обозначать штрихом.

Пусть на пленку падают с обеих сторон когерентные монохроматические плоские электромагнитные волны, причем угол падения в каждой среде равен углу преломления (см. рис. 2). В каждой среде будут тогда прямая и обратная волны. Обобщенные амплитуды 


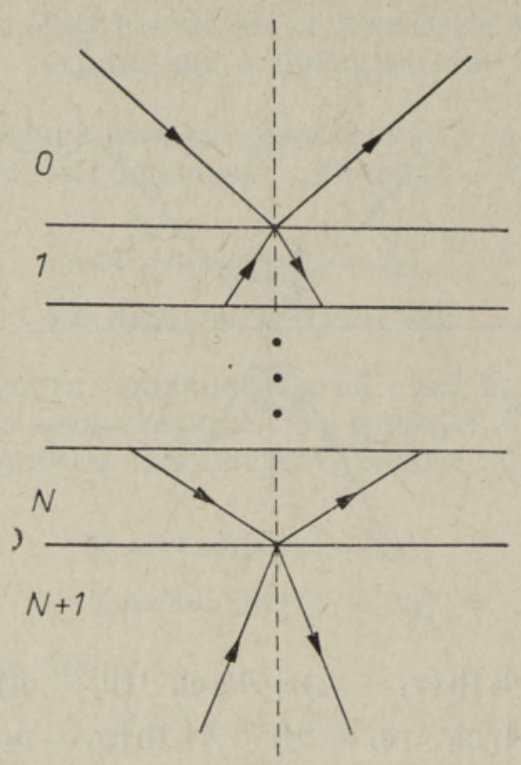

Рис. 2.

этих волн в начале $m$-й среды (т. е. на границе ее с $(m-1)$-й средой) обозначим через $A_{m}$ и $A_{m}^{\prime}(m>0)$, а через $A_{0}$ и $A_{0}^{\prime}$ обозначим амплитуды в 0 -й среде на ее границе с 1-й средой. Введем также матрицы

$$
\mathrm{A}_{m}=\left(\begin{array}{l}
A_{m} \\
A_{m}^{\prime}
\end{array}\right),-m=0,1, \ldots N+1
$$

Тогда, согласно формуле (29),

$$
\mathrm{A}_{m}=M\left(\alpha_{m}\right) G\left(v_{m}-v_{m+1}\right) \mathrm{A}_{m+1}
$$

где

$$
M(\alpha)=\left(\begin{array}{cc}
e^{i \alpha} & 0 \\
0 & e^{-i \alpha}
\end{array}\right)
$$

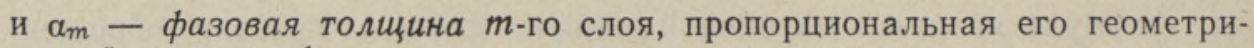
ческой толщине $h_{m}$ :

$$
\alpha_{m}=(\omega / c) n_{m} h_{m} \cos \vartheta_{m} .
$$

В общем случае фазовая толщина комплексна. Обозначив еще для краткости

$$
v_{m}-v_{m+1}=v_{m, m+1}
$$

и распространив формулу (33) на всю пленку, находим

$$
\mathrm{A}_{0}=G\left(v_{01}\right) M\left(\alpha_{1}\right) G\left(v_{12}\right) M\left(\alpha_{2}\right) \ldots M\left(\alpha_{N}\right) G\left(v_{N, N+1}\right) A_{N+1} .
$$

Эта формула является обобщением формул Френеля с простой границы раздела двух сред на $N$-слойную пленку. Обозначив

$$
F=G\left(v_{01}\right) M\left(\alpha_{1}\right) G\left(v_{12}\right) M\left(\alpha_{2}\right) \ldots M\left(\alpha_{N}\right) G\left(v_{N, N+1}\right),
$$

имеем

$$
\mathrm{A}_{0}=F \mathrm{~A}_{N+1}
$$

Очевидно,

$$
\|F\|=1 \text {. }
$$




\section{Линейные коэффициенты пленки}

Матрица $F$ имеет простой физический смысл. Она преобразует амплитуды прямой и обратной волн при прохождении сквозь пленку. Выясним смысл отдельных элементов этой матрицы.

Для этого введем прежде всего понятие сопряженной системы. Это - система, отличающаяся от данной только тем, что проницаемости всех сред и все углы заменены комплексно-сопряженными величинами. Очевидно, формулы Френеля остаются при переходе к сопряженной системе неизменными, поскольку они по виду не зависят от конкретных значений проницаемостей, а элементарный закон преломления Снеллиуса остается в силе.

Применив формулу (38) к сопряженной системе, находим

$$
\tilde{F}=G\left(\tilde{v}_{01}\right) M\left(\tilde{\alpha}_{1}\right) G\left(\tilde{v}_{12}\right) M\left(\tilde{\alpha}_{2}\right) \ldots M\left(\tilde{\alpha}_{N}\right) G\left(\tilde{v}_{N, N+1}\right),
$$

где $\sim$ есть знак сопряжения. Но так как, согласно формулам (6), (34) и (35),

$$
\begin{aligned}
& \tilde{v}=v^{*}, \\
& \tilde{\alpha}=\alpha^{*},
\end{aligned}
$$

то, перейдя в формуле (41) к комплексно-сопряженным значениям, находим

$$
F^{*}=G\left(v_{01}\right) M\left(-\alpha_{1}\right) G\left(v_{12}\right) M\left(-\alpha_{2}\right) \ldots M\left(-\alpha_{N}\right) G\left(v_{N, N+1}\right) .
$$

Отсюда

$$
F^{*-1}=G\left(v_{N+1, N}\right) M\left(\alpha_{N}\right) \ldots M\left(\alpha_{2}\right) G\left(v_{21}\right) M\left(\alpha_{1}\right) G\left(v_{10}\right),
$$

T. e.

$$
F^{*-1}=F^{\prime} .
$$

Иначе можно матрицу $F^{\prime}$ выразить следующим образом. Из формулы (39) следует

$$
\mathrm{A}_{N+1}=F^{-1} \mathrm{~A}_{0} \text {. }
$$

Но так как для обратного направления прямая волна является обратной и наоборот, то

$$
\begin{gathered}
\mathrm{A}^{\prime}{ }_{N+1}=\left(\begin{array}{ll}
0 & 1 \\
1 & 0
\end{array}\right) \mathrm{A}_{N+1}, \\
\mathrm{~A}_{0}^{\prime}=\left(\begin{array}{ll}
0 & 1 \\
1 & 0
\end{array}\right) \mathrm{A}_{0} .
\end{gathered}
$$

Следовательно,

$$
\mathrm{A}^{\prime}{ }_{N+1}=\left(\begin{array}{ll}
0 & 1 \\
1 & 0
\end{array}\right) F^{-1}\left(\begin{array}{ll}
0 & 1 \\
1 & 0
\end{array}\right) \mathrm{A}_{0}^{\prime}
$$

T. e.

$$
F^{\prime}=\left(\begin{array}{ll}
0 & 1 \\
1 & 0
\end{array}\right) F^{-1}\left(\begin{array}{ll}
0 & 1 \\
1 & 0
\end{array}\right)
$$

Сопоставляя эту формулу с формулой (45), находим

$$
\tilde{F}^{*}=\left(\begin{array}{ll}
0 & 1 \\
1 & 0
\end{array}\right) F\left(\begin{array}{ll}
0 & 1 \\
1 & 0
\end{array}\right),
$$

откуда 


$$
\begin{aligned}
& F_{11}^{*}=F_{22}, \\
& F_{22}^{*}=F_{11}, \\
& F_{12}^{*}=F_{21}, \\
& F_{21}^{*}=F_{12},
\end{aligned}
$$

в силу чего, согласно формуле (40),

$$
F_{11} \widetilde{F}_{11}^{*}-F_{21} \widetilde{F}_{21}^{*}=1 .
$$

Теперь легко найти смысл элементов матрицы $F$. Из формулы (39) следует

$$
\begin{aligned}
& A_{0}=F_{11} A_{N+1}+F_{12} A^{\prime}{ }_{N+1}, \\
& A^{\prime}{ }_{0}=F_{21} A_{N+1}+F_{22} A^{\prime}{ }_{N+1} .
\end{aligned}
$$

С другой стороны, если $r$ и $t-$ амплитудные коэффициенты отражения и пропускания пленки, то

$$
\begin{aligned}
& A_{0}^{\prime}=r A_{0}+t^{\prime} A^{\prime}{ }_{N+1}, \\
& A_{N+1}=t A_{0}+r^{\prime} A^{\prime}{ }_{N+1} .
\end{aligned}
$$

Выражая из этих формул $A_{0}$ и $A^{\prime}{ }_{0}$, находим

$$
\begin{aligned}
& A_{0}=t^{-1} A_{N+1}-\left(r^{\prime} / t\right) A_{N+1}^{\prime}, \\
& A_{0}^{\prime}=(r / t) A_{N+1}+\left(t^{\prime}-r r^{\prime} / t\right) A_{N+1}^{\prime} .
\end{aligned}
$$

Сравнение с формулами (53) дает

$$
\begin{aligned}
& F_{11}=t^{-1}, \\
& F_{21}=r / t, \\
& F_{12}=-r^{\prime} / t, \\
& F_{22}=t^{\prime}-r r^{\prime} / t .
\end{aligned}
$$

Первые две формулы можно переписать в виде

$$
\begin{aligned}
& F_{11}=b, \\
& F_{21}=a,
\end{aligned}
$$

где $a$ и $b$ - линейные коэффициенты пленки, определяемые формулой (21). Для элементов второго столбца матрицы $F$ согласно формулам (51) имеем

$$
\begin{aligned}
& F_{22}=\tilde{b}^{*}, \\
& F_{12}=\tilde{a}^{*},
\end{aligned}
$$

так что вся матрица имеет вид

$$
F=\left(\begin{array}{ll}
b & \tilde{a}^{*} \\
a & \tilde{b}^{*}
\end{array}\right)
$$

причем

$$
b \tilde{b}^{*}-a \tilde{a}^{*}=1 .
$$

Остальные две формулы (56) определяют $a^{\prime}$ и $b^{\prime}$. Сначала имеем 


$$
\begin{aligned}
& \tilde{a}^{*}=-r^{\prime} \mid t, \\
& \tilde{b}^{*}=t^{\prime}-r r^{\prime} \mid t .
\end{aligned}
$$

Подставляя эти выражения вместе с выражениями $b=1 / t$ и $a=r / t$ в формулу (60), находим $t^{\prime}=t$, т. е.

$$
b^{\prime}=b \text {. }
$$

Следовательно, согласно первой формуле (61),

$$
a^{\prime}=-\tilde{a}^{*} \text {. }
$$

Для простой границы раздела двух сред $\tilde{a}^{*}=a$ и $\tilde{b}^{*}=b$, так что формулы (60) и (63) принимают вид $a^{\prime}=-a$ и $b^{2}-a^{2}=1$, в согласии с формулами (22) и (24).

Если пленка ограничена средами с вещественными значениями $\varepsilon, \mu$ и $\vartheta$, то линейные коэффициенты определяют энергетические коэффициенты отражения и пропускания пленки по формулам

$$
\begin{aligned}
& R=a a^{*} / b b^{*}, \\
& T=1 / b b^{*} ;
\end{aligned}
$$

являющимся обобщением формул (23) для простой границы раздела.

Выведем в заключение еще одну простую формулу для матрицы $F$ и ее элементов. Если выберем произвольно какой-либо слой, назвав его промежуточным слоем, то пленка окажется разделенной этим слоем на две части. Обозначим их индексами 1 и 2 , а промежуточный слой - индексом $m$. Тогда из формулы (38) вытекает

$$
F=F_{1} M\left(\alpha_{m}\right) F_{2},
$$

где $F_{1}$ и $F_{2}$ относятся к двум частям пленки при условии, что промежуточный слой является конечной средой для первой части и начальной для второй. Для элементов мартиц $F_{1}, F_{2}$ и $F$ получаем отсюда согласно формулам (34) и (59) следующие соотношения

$$
\begin{aligned}
& a=a_{1} b_{2} e^{i \alpha_{m}}+a_{2} \widetilde{b}_{1}^{*} e^{-i \alpha_{m}}, \\
& b=b_{1} b_{2} e^{i \alpha_{m}}+a_{2} \tilde{a}_{1}^{*} e^{-i \alpha_{m}} .
\end{aligned}
$$

Эти формулы показывают, что $a$ и $b$ зависят линейно как от $a_{1}, b_{1}$, $\tilde{a}_{1}{ }^{*}, \tilde{b}_{1}{ }^{*}$, так и от $a_{2}, b_{2}$. Это свойство оправдывает данное величинам $a$ и $b$ название линейных коэффициентов.

\section{Л И Т Е Р А Т Р А}

1. Кард П. Г. Анализ и синтез многослойных интерференционных пленок. Таляин, «Валгус», 1971.

Тартуский государственный университет
Поступила в редакцию 27/II 1984 


\section{UUEKUJULISED FRESNELI VALEMID}

Fresneli valemid elektromagnetlaine peegeldumise ja murdumise kohta kahe keskkonna lahutuspinnal, üldistatuna kahe koherentse laine kahelt poolt langemise juhule, on maatrikskujus (29) ehk (31), kus $G$ ja A on valemitega (27) ja (28) defineeritud maatriksid, indeksid 1 ja 2 tähistavad kaht keskkonda, $A$ on otsesuunalise $(1 \rightarrow 2)$ ja $A^{\prime}$ vastassuunalise $(2 \rightarrow 1)$ laine valemiga (7) defineeritud üldistatud amplituid; suurused $v$ (refraktorid) on defineeritud valemitega (6). Valemites (6) ja (7) on $\varepsilon_{0}$ ja $\mu_{0}$ elektriline ja magnetiline konstant, $\varepsilon$ ja $\mu$ keskkonna suhteline elektriline ja magnetiline läbitavus, $n$ murdumisnäitaja, $\theta$ langemis- või murdumisnurk, $E$ ja $H$ elektri- ja magnetvälja tugevuse amplituudid. Uuekujuliste Fresneli valemite rakendamine mitmekihilisele interferentskilele annab kergesti kile pöhivalemi (39), kus maatriks $F$ on defineeritud valemiga (38) ja selles esinevad suurused valemitega (34)-(36). Maatriksi $F$ esimese veeru elemendid (valem (59)) on kile lineaartegurid $a$ ja $b$ (vt. valem (21), kus $r$ ja $t$ on kile amplituudsed peegeldumis- ja läbilaskvustegurid). $F$-i teise veeru elemendid on kaaskile lineaartegurite kaaskompleksid $\tilde{a}^{*}$ ja $\tilde{b}^{*}$, kusjuures kaaskile erineb antud kilest ainult selle poolest, et kōikide keskkondade läbitavused ja langemis- (vōi murdumis-) nurgad on kaaskomplekssete väärtustega.

\section{P. KARD}

\section{A NEW FORM OF THE FRESNEL FORMULAE}

The well-known Fresnel formulae for the reflection and refraction of the electromagnetic waves, provided that coherent waves are incident from both media, are presented in a simple matrix form, viz. formulae (29) or (31), where $G$ and A are defined by the formulae (27) and (28); the indices 1 and 2 denote two media; $A$ and $A^{\prime}$ are the generalized amplitudes ( $\mathrm{s}$. the formula $(7)$ ) of the direct $(1 \rightarrow 2)$ and the backward $(2 \rightarrow 1)$ wave; the quantities $v$ (refractors) are defined by the formula (6). In the formulae (6) and (7) $\varepsilon_{0}$ an $\mu_{0}$ are the electric and magnetic constants, $\varepsilon$ is the relative permittivity and $u$ the relative permeance of the medium, $\theta$ is the angle of incidence or refraction, $E$ and $H$ are the amplitudes of the electric and magnetic intensities. This new representation of the Fresnel formulae leads directly to the formula (37), which fully accounts for the optical properties of the multilayer interference films. The quantities entering this formula are defined by the formulae $(34)-(36)$. The elements of the first column of the matrix $F$ (s. the formulae (38) and (59)) are the linear coefficients $a, b$ of the film (s. formula (21), where $r$ and $t$ are the amplitude reflectance and amplitude transmittance). The elements of the second column are the complex conjugated values of the linear coefficients of the conjugated film, having complex conjugated values of the permittivities and the permeances of all media and of all angles of incidence and refraction. 